\title{
Evaluation of the Single-Port Laparoscopic Right Hemicolectomy Learning Curve
}

\author{
Virgilio V. George", Michael J. Guzman, Joshua A. Waters, Andrea L. Jester, \\ Don J. Selzer, Bruce W. Robb \\ Department of Surgery, Indiana University School of Medicine, Indianapolis, USA \\ Email: "vigeorge@iupui.edu
}

Received June 17, 2013; revised July 16, 2013; accepted July 24, 2013

Copyright (c) 2013 Virgilio V. George et al. This is an open access article distributed under the Creative Commons Attribution License, which permits unrestricted use, distribution, and reproduction in any medium, provided the original work is properly cited.

\begin{abstract}
Background: The use of single-port laparoscopy has gained popularity within recent years. Part of the appeal in learning this approach is that it draws heavily from concepts mastered through conventional laparoscopy. Various studies have shown the efficacy and feasibility of the single-port laparoscopic approach, but there are few that examine the learning curve in adopting this new technique. Objective: Our goal was to better define the learning curve in performing a single-port laparoscopic right hemicolectomy. Design: A review of prospectively gathered operative data was performed to analyze the results of single-port laparoscopic right hemicolectomies performed within our institution by experienced laparoscopic surgeons. The first 100 cases were divided into quintiles. Comparisons were made among the cohorts regarding patient demographics, operative time, length of stay, conversions, and complications. Results: There was no difference among quintiles with regard to age, sex, BMI, or ASA class. Operative time, conversions, length of stay, and number of complications did not significantly vary among each group of patients. There was a significant difference in estimated blood loss and length of stay between the fifth cohort and the others due to one patient's poor outcome. Conclusions: The single-port laparoscopic right hemicolectomy learning curve for surgeons already skilled in laparoscopy is short. There are few differences in various outcome measures among groups at any stage in the learning curve. The skills utilized to perform conventional laparoscopic colorectal surgery readily translate to the single-port approach and result in proficiency from nearly the start.
\end{abstract}

Keywords: Single-Port; Laparoscopy; Learning Curve; Colectomy

\section{Introduction}

New surgical techniques are constantly being developed around the world. Some interventions never gain traction, while others become an important part of a surgeon's skill set. Single-port laparoscopy is one approach that has steadily gained popularity across various surgical disciplines and is a frequent topic of investigation. It would appear that single-port laparoscopy will remain a part of surgical therapy for some time.

As the field of single-port laparoscopy grows, the trend in publications changes with it. To date, there have been a number of studies from the general, urologic, and gynecologic surgery literature looking at the feasibility and safety of the single-port laparoscopic approach to

\footnotetext{
* Selected for podium presentation at the American Society of Colon and Rectal Surgeons annual scientific meeting San Antonio, TX June 2-6, 2012.

\#Corresponding author.
}

various surgeries [1-5]. Although the technique may vary, it would appear that nearly any surgery that can be done laparoscopically can also be done using a single-port approach.

Colon and rectal surgery is no stranger to this trend and in recent years it has seen a vast increase in publications about the use of single-port laparoscopy. Our group has published the largest series of single-port laparoscopic right hemicolectomies to date [6].

When learning any new procedure, there is a learning curve that each person must complete in order to become proficient. There has been some data addressing the learning curve for single-port laparoscopic cholecystectomy, gastric band placement, and nephrectomy [7-9]. However, there have been no studies looking at the learning curve for performing any single-port laparoscopic colorectal surgeries. In this study, we aim to define the learning curve for single-port laparoscopic right hemicolectomy (SPLRH). 


\section{Materials and Methods}

\subsection{Patient Selection}

All study participants were selected based on the guidelines and regulations set by the Indiana University School of Medicine Institutional Review Board.

This series represents a retrospective analysis of prospectively gathered data from a consecutive series of patients undergoing planned SPLRH in a single colorectal surgery group between January 2008 and November 2010. The primary inclusion criterion was planned single port laparoscopic approach in the setting of a right hemicolectomy. Patients were included in this series regardless of operative indication, urgency of operation, or ultimate approach (i.e. conversion to open). Patients were then divided into quintiles of twenty consecutive cases for the purpose of statistical analysis.

\subsection{Measurements and Endpoints Assessed}

The data collected and analyzed in this series fall into three groups: patient demographics, operative measurements, and short term outcomes. Patient specific data included: gender, age, body mass index (BMI), and American Society of Anesthesiology (ASA) classification as a surrogate of patient comorbidity. The operative measurements used in our study were: operative time, conversions to either multi-port laparoscopy or open surgery, and the estimated blood loss (EBL). Short term outcome measurements were total length of stay (LOS) and number of complications. The different complications measured within our study included superficial wound infection, abdominal abscess, anastomotic leak, renal failure defined by increase in creatininegreater than or equal to $1 \mathrm{mg} / \mathrm{dL}$ above baseline, post-operative ileus, post-operative bleeding (regardless of intervention), and 30 day mortality.

\subsection{Operative Technique}

The operative technique used by the surgeons within our institution has previously been described by Waters et al. $[6,10]$. Briefly, a vertical incision was made in the fascia large enough to accommodate the single-port trocar. A standard 5-mm $30^{\circ}$ laparoscope was then inserted (or rarely a $10-\mathrm{mm}$ laparoscope), followed by $25-\mathrm{mm}$ working ports with non-articulating instruments. The ileocolic pedicle was then elevated in order to dissect the colon off its retroperitoneal attachments and duodenum in a medial to lateral fashion. Once this was complete, the ileocolic vessels were divided using an energy device. The hepatic flexure and the lateral attachments were then taken down from superior to inferior. Fascial incisions were enlarged as necessary to exteriorize the specimen for division and anastomosis. After inspecting the anastomosis intracor- poreally, the fascia was closed in either a running or figure-of-eight fashion.

\subsection{Data Analysis and Presentation}

All data was compiled, analyzed, and formatted into figures using GraphPad Prism (La Jolla, CA). Continuous variables were compared using one-way analysis of variance (ANOVA). This data was described using means with ranges where appropriate. Categorical data was compared using a Chi-square test and presented as proportions or number of instances where appropriate. Probability values less than or equal to 0.05 were considered statistically significant. Data tables were made using Microsoft Word 2010 (Redmond, WA).

\section{Results}

During our study period, a total of 100 patients underwent SPLRH by our group of surgeons. The patient demographics for all patients included in our study are outlined in Table 1. As outlined in the table, there was no statistical difference between quintiles with regards to patient gender, age, ASA classification, or BMI. When considering the entire study population, $61 \%$ were male, the mean age was 64 years, the mean ASA class was 3, and mean BMI was 28.

Operative measurements are described in Table 2 . Operative times are reported as mean time in minutes with range, and mean time of operation throughout the study period was 114 minutes with a range from 64 - 270 minutes. Conversion to either multi-port laparoscopy or open was made at the discretion of each surgeon. Indications for conversion varied from failure to progress, discovery of more invasive cancer than anticipated preoperatively, and inability to control bleeding. Estimated blood loss is displayed in more detail in Figure 1. The fifth quintile had a significantly higher mean blood loss (228 $\mathrm{mL}$ ) compared to the other groups. This quintile contained one patient who was a particularly high outlier in operative measures and short term outcomes.

Short term outcomes are displayed in Table 3. The mean LOS for the entire population was 5.6 days with a

Table 1. Patient demographics.

\begin{tabular}{ccccc}
\hline $\begin{array}{c}\text { Patient } \\
\text { group }\end{array}$ & $\%$ male & $\begin{array}{c}\text { Age (mean } \\
\text { years, } \\
\text { range) }\end{array}$ & $\begin{array}{c}\text { ASA class } \\
\text { (mean, } \\
\text { range) }\end{array}$ & $\begin{array}{c}\text { BMI (mean, } \\
\text { range) }\end{array}$ \\
\hline $1-20$ & 55 & $65(39-90)$ & $3(1-3)$ & $28(20-39)$ \\
$21-40$ & 65 & $65(25-85)$ & $3(2-4)$ & $27(21-46)$ \\
$41-60$ & 55 & $67(26-86)$ & $3(2-4)$ & $26(18-36)$ \\
$61-80$ & 70 & $64(30-85)$ & $3(2-4)$ & $29(19-41)$ \\
$81-100$ & 65 & $56(28-83)$ & $3(2-4)$ & $30(18-43)$ \\
\hline
\end{tabular}


Table 2. Operative measures.

\begin{tabular}{cccc}
\hline $\begin{array}{c}\text { Patient } \\
\text { group }\end{array}$ & $\begin{array}{c}\text { Mean operative } \\
\text { time (min) }\end{array}$ & $\begin{array}{c}\text { Operative time } \\
\text { ranges (min) }\end{array}$ & Conversions \\
\hline $1-20$ & 109 & $71-212$ & 1 open \\
$21-40$ & 119 & $83-194$ & 2 lap, 1 open \\
$41-60$ & 108 & $64-177$ & 1 open \\
$61-80$ & 112 & $67-190$ & 0 \\
$81-100$ & 127 & $86-270$ & 1 open \\
\hline
\end{tabular}

Table 3. Short term outcomes.

\begin{tabular}{cccc}
\hline $\begin{array}{c}\text { Patient } \\
\text { group }\end{array}$ & $\begin{array}{c}\text { Mean length of } \\
\text { stay (days) }\end{array}$ & $\begin{array}{c}\text { Length of stay } \\
\text { range (days) }\end{array}$ & $\begin{array}{c}\text { Complications } \\
\text { (\#) }\end{array}$ \\
\hline $1-20$ & 6 & $2-24$ & 4 \\
$21-40$ & 4 & $2-11$ & 3 \\
$41-60$ & 5 & $2-10$ & 1 \\
$61-80$ & 5 & $2-26$ & 3 \\
$81-100$ & $7^{*}$ & $2-48$ & 2 \\
\hline
\end{tabular}

${ }^{*} \mathrm{p}<0.05$.

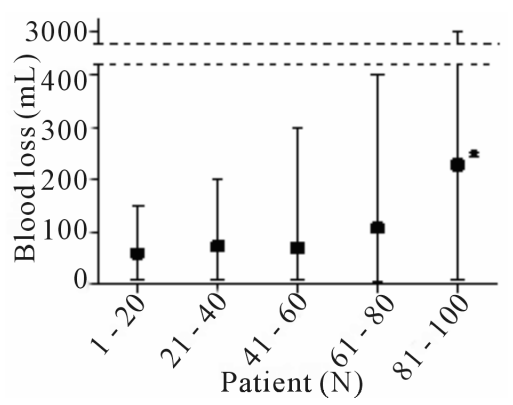

Figure 1. Estimated blood loss (mean \pm range) by 20 patient cohorts. "Significantly different from other groups (p < 0.05).

range from 2 - 48 days. Here again, the high outlier in the fifth quintile made it significantly different from the other groups. This patient had the longest stay out of the entire population and eventually died due to multi-system organ failure. When comparing the number of complications between each quintile, there was no difference between groups and overall rate of morbidity was $13 \%$.

\section{Discussion}

Single-port laparoscopy is becoming a popular option in the field of colorectal surgery. However, because it is a relatively new approach many surgeons do not have any formal training in performing these operations. Those who are taking it upon themselves to learn this new technique do not yet know the number of cases it takes to become proficient in safely performing a SPLRH, and our study sought to evaluate the learning curve for this operation. We have found that the learning curve is quite short and almost non-existent for those already skilled in conventional laparoscopic surgery and more specifically, right hemicolectomy.

When examining the patients included in this study it is important to note that our population is a fairly accurate representation of a typical surgeon's patient mix, as we did not select out patients that are particularly slender, young, or healthy. In fact, the average patient who underwent SPLRH in our series was overweight (mean BMI 28), older (mean age 64), and with significant comorbid conditions (mean ASA 3). This makes our results easier to reproduce by the average colorectal surgeon in practice.

Our group has been slowly expanding inclusion criteria for those who are offered a SPLRH as experience grows and currently any patient who would be an appropriate candidate for conventional laparoscopy is also considered appropriate for the single-port approach, and they are offered that option as well. It is likely that as the general public becomes more aware of single-port laparoscopy the demand for it will increase based on its potential benefits whether perceived or truly present.

With respect to operative times, we did not find any significant change in the duration of operation between quintiles. These times are also similar to those for performing a conventional laparoscopic right hemicolectomy. While this could mean that we have not yet started the drastic improvement phase of the typical learning curve, we think that is unlikely. All surgeons within our group have significant experience with conventional laparoscopy from formal fellowship training and years of practice as attending surgeons. While it is possible that at some point SPLRH could be performed faster than conventional laparoscopy, our operative times are already similar to other groups with right hemicolectomy [11]. It is likely that the skills already established with multi-port laparoscopic right hemicolectomy readily translate the single-port approach. This may be due in part to our use of standard laparoscopic instruments and cameras, which eliminates the learning of new specialized and more complicated instrumentation.

Conversions from the single-port approach to either a multi-port approach or open technique did not change significantly throughout our study period. Interestingly, the only two conversions to conventional laparoscopy took place within the second quintile by a single surgeon who did so because of failure to progress within the operation from difficult dissection. The conversions to open surgery were done because of difficult dissection, where it was believed that additional trocars would not significantly improve the chance of completing the operation laparoscopically and in the final instance, significant 
bleeding was encountered that required direct visualization for control. However, our overall conversion rate was in line with other reported experiences [11]. Additionally, we believe that conversion of the procedure from a single-port approach should not be deemed a failure as much as it represents better judgment on the part of the surgeon to change direction when the individual scenario requires it.

Measurement of blood loss during an operation can be interpreted in various ways. A large amount of blood loss to one surgeon may mean that the surgery was particularly difficult and that it may be expected that the patient has a higher risk of having a post-operative complication, but another surgeon may see that same blood loss and interpret it as poor performance of the operating surgeon. With that in mind, there was no difference in mean blood loss across the quintiles until the final group. As previously mentioned, this was due to one particular patient in that group who had a significant blood loss due to injury to the middle colic vein. Interestingly, as the series went on, there was an increase in the range of blood loss.

Our short term outcomes with regard to length of stay and peri-operative morbidity did not change through the progression of cases. Rather, both remained consistent with other reported series of both single-port and multiport laparoscopic colectomies [10,12-14]. This suggests that the key to determining patient recovery may not lie within the approach between single or multi-port laparoscopy, and instead hinders on the nature of the operation itself and/or inherent patient factors. Importantly, the use of the single-port approach did not negatively impact the rates of post-operative morbidity.

When examining the data presented here, it is important to take into account a few limitations of this study. These cases were performed by multiple surgeons in a group. However, one surgeon either performed or assisted in the majority of the cases examined in this series, and all of our surgeons have similar training, experience with colorectal surgery, and method to performing a SPLRH, reducing the inter-surgeon variability. We did look at the data from a single surgeon's perspective but did not find significant difference between his and the rest of the group's measures. Another limitation is that the role of residents and fellows cannot be quantified within the data. However, as would be expected, there is a graded role of involvement over time with experience by both the trainee and staff surgeon.

The introduction of new surgical techniques requires completing a number of procedures before becoming proficient. With the increasing presence of single-port laparoscopy within surgical practice, it is important to understand how long it takes a surgeon to become facile with this evolving approach. Prior studies have already examined the learning curve for various single-port laparoscopic operations [7-9], and have concluded that the learning curve is fairly short for those already experienced in their field. However, this is the first report examining the learning curve with regards to colorectal surgery. Whether this approach will become a part of an average colorectal surgeon's practice has yet to be determined, and further investigations to understand its potential long term benefits and hazards are needed.

\section{Conclusion}

The learning curve for SPLRH is short for colorectal surgeons who already experienced with conventional laparoscopic operations. Surgeons who desire to start offering their patients a single-port approach to right hemicolectomy can do so without significant increase in operative time, length of stay, or per-operative morbidity. Surgeons will need to become facile with SPLRH to keep up with patients' increasing awareness and desire for this approach.

\section{REFERENCES}

[1] P. Bucher, F. Pugin and P. Morel, "Single Port Access Laparoscopic Right Hemicolectomy,” International Journal of Colorectal Disease, Vol. 23, No. 10, 2008, pp. 1013-1016.

http://dx.doi.org/10.1007/s00384-008-0519-8

[2] A. N. Fader and P. F. Escobar, "Laparoendoscopic Single-Site Surgery (LESS) in Gynecologic Oncology: Technique and Initial Report," Gynecologic Oncology, Vol. 114, No. 2, 2009, pp. 157-161. http://dx.doi.org/10.1016/j.ygyno.2009.05.020

[3] G. Navarra, E. Pozza, S. Occhionorelli, P. Carcoforo and I. Donini, "One-Wound Laparoscopic Cholecystectomy," British Journal of Surgery, Vol. 84, No. 5, 1997, p. 695. http://dx.doi.org/10.1002/bjs.1800840536

[4] L. E. Ponsky, E. E. Cherullo, M. Sawyer and D. Hartke, "Single Access Site Laparoscopic Radical Nephrectomy: Initial Clinical Experience,” Journal of Endourology, Vol. 22, No. 4, 2008, pp. 663-666. http://dx.doi.org/10.1089/end.2007.0427

[5] A. A. Saber, T. H. El-Ghazaly and D. B. Minnick, "Single Port Access Transumbilical Laparoscopic Roux-en-Y Gastric Bypass Using the SILS Port: First Reported Case,” Surgical Innovation, Vol. 16, No. 4, 2009, pp. 343-347. http://dx.doi.org/10.1177/1553350609354604

[6] J. A. Waters, B. M. Rapp, M. J. Guzman, A. L. Jester, D. J. Selzer, B. W. Robb, B. J. Johansen, B. M. Tsai, D. C. Maun and V. V. George, "Single-Port Laparoscopic Right Hemicolectomy: The First 100 Resections,” Diseases of the Colon \& Rectum, Vol. 55, No. 2, 2012, pp. 134-139. http://dx.doi.org/10.1097/DCR.0b013e31823c0ae4

[7] M. Gawart, S. Dupitron and R. Lutfi, "Laparoendoscopic Single-Site Gastric Bands versus Standard Multiport Gastric Bands: A Comparison of Technical Learning Curve Measured by Surgical Time," The American Journal of Surgery, Vol. 203, No. 3, 2012, pp. 327-329. 
http://dx.doi.org/10.1016/j.amjsurg.2011.10.001

[8] J. Hernandez, S. Ross, C. Morton, K. McFarlin, S. Dahal, F. Golkar, M. Albrink and A. Rosemurgy, "The Learning Curve of Laparoendoscopic Single-Site (LESS) Cholecystectomy: Definable, Short, and Safe,” Journal of the American College of Surgeons, Vol. 211, No. 5, 2010, pp. 652-657.

http://dx.doi.org/10.1016/j.jamcollsurg.2010.07.008

[9] L. Wang, B. Liu, Z. Wu, Q. Yang, M. Hehir, W. Chen, Z. $\mathrm{Xu}$, L. Xiao, F. Wang and Y. Sun, "Transumbilical Laparoendoscopic Single-Site Surgery: More than 1-Year Experience in Radical Nephrectomy and Its Learning Curve Study," Journal of Endourology, Vol. 25, No. 2, 2011, pp. 1859-1865.

http://dx.doi.org/10.1089/end.2011.0015

[10] J. A. Waters, M. J. Guzman, A. D. Fajardo, D. J. Selzer, E. A. Wiebke, B. W. Robb and V. V. George, "SinglePort Laparoscopic Right Hemicolectomy: A Safe Alternative to Conventional Laparoscopy," Diseases of the Colon \& Rectum, Vol. 53, No. 11, 2010, pp. 1467-1472. http://dx.doi.org/10.1007/DCR.0b013e3181f23ca0
[11] T. Makino, J. W. Milsom and S. W. Lee, "Feasibility and Safety of Single-Incision Laparoscopic Colectomy: A Systematic Review," Annals of Surgery, Vol. 255, No. 4, 2012, pp. 667-676.

http://dx.doi.org/10.1097/SLA.0b013e31823fbae7

[12] The Clinical Outcomes of Surgical Therapy Study Group, "A Comparison of Laparoscopically Assisted and Open Colectomy for Colon Cancer,” The New England Journal of Medicine, Vol. 350, 2004, pp. 2050-2059. http://dx.doi.org/10.1056/NEJMoa032651

[13] B. A. Boone, P. Wagner, E. Ganchuk, L. Evans, H. J. Zeh, D. L. Bartlett and M. P. Holtzman, "Single-Incision Laparoscopic Right Colectomy in an Unselected Patient Population,” Surgical Endoscopy, Vol. 26, No. 6, 2011, pp. 1595-1601.

[14] A. J. Senagore and C. P. Delaney, “A Critical Analysis of Laparoscopic Colectomy at a Single Institution: Lessons Learned after 1000 Cases," The American Journal of Surgery, Vol. 191, No. 3, 2006, pp. 377-380. http://dx.doi.org/10.1016/j.amjsurg.2005.10.039 